\title{
EL DIBUJO TÉCNICO MANUAL Y SU VIGENCIA EN EL ACTUAL CONTEXTO TECNOLÓGICO
}

\author{
Technical drawing and its validity in the current technological context
}

GIOVANY SUÁREZ PINILLA ${ }^{1}$

Recibido:22 de agosto de 2019. Aceptado:10 de diciembre de 2019

DOI: http://dx.doi.org/10.21017/rimci.2020.v7.n13.a76

\begin{abstract}
Resumen
Desde la antigüedad, el dibujo se ha utilizado para expresar las ideas artísticas y científicas creadas en el cerebro humano. Aunque la realización de planos y bosquejos -también conocido como dibujo técnico hecho a mano- es un tema tradicional que se enseña en las universidades para programas de ingeniería y arquitectura, las tendencias actuales tienden a reemplazarla por software de dibujo asistido por computador. Aunque las nuevas tecnologías, en particular las TIC (tecnologías de la información y la comunicación) apoyan con una gran cantidad de ventajas el proceso de aprendizaje, el dibujo a mano alzada también proporciona a los estudiantes habilidades muy poderosas que serán del mayor provecho en su formación y su vida laboral. A lo largo de los siguientes párrafos, el autor presenta una breve revisión sobre la relación existente entre dibujo y ciencia, así como el importante papel que juega el dibujo a mano alzada en los tiempos actuales.
\end{abstract}

Palabras clave. Dibujo técnico; Bosquejo; CAD; Aprendizaje significativo; TICs; educación.

\begin{abstract}
From ancient times, drawing has been used to express both the artistic and scientific ideas created in human brain. Although drafting -otherwise known as technical drawing made by hand-is a traditional subject taught at universities for engineering and architectural programs, current trends tend to replace it for computer assisted drawing software. Yet new technologies, in particular ICTs (information and communication technology) support with a large number of advantages the learning process, freehand drawing also provide students with very powerful skills that will be of their greatest benefit at school and work. Through the following paragraphs, the author presents a brief review about the relationship between drawing and science as well as the significant role that freehand drawing plays in the current times.
\end{abstract}

Key words. Technical drawing; drafting; CAD; meaningful learning; ICTs; education.

\section{INTRODUCCIÓN}

L A FORMACIÓN académica dentro de las aulas de clase, no puede ser ajena a los continuos avances tecnológicos que a pasos agigantados experimentamos hoy día; aplicaciones móviles, tabletas digitales y un gran número de paquetes informáticos, ofrecen a estudiantes y docentes múltiples herramientas para el diseño y la expresión gráfica en diversos campos del conocimiento. No obstante, en la atmósfera digital actual, vale la pena preguntarse si aún tiene vigencia el aprendizaje de las técnicas clásicas del dibujo técnico, aquella $\mathrm{s}$ basadas en el uso de instrumentos tales como la regla, el compás, los lápices y las plantillas para letra técnica. Parece que ya son cosa del pasado los amplios salones de dibujo con grandes mesas de madera y el rigor en la impecabilidad y pulcritud de una plancha hecha a mano. Sin embargo, no pueden menospreciarse las aptitudes y cualidades que despierta en los estudiantes este tipo de prácticas. Más bien, urge retomar estas cáte-

1 Ingeniero Civil y Magíster en Ingeniería - Universidad Nacional de Colombia. Estudios de Posgrado en Docencia en la Universidad de la Sabana y la Fundación Universitaria Monserrate. Graduado en 1994 como Dibujante Técnico Industrial del Instituto Técnico Central La Salle. Correo electrónico: gsuarez@urepublicana.edu.co jgsuarezp@unal.edu.co 
dras en las facultades de ingeniería y arquitectura, porque los profesionales en formación requerirán en su vida profesional, tarde o temprano, los conceptos espaciales y geométricos involucrados en la expresión gráfica, especialmente, en un mundo saturado de herramientas tecnológicas que no pocas veces dejan sin lugar a la abstracción y a la creatividad humana.

\section{Contexto Histórico}

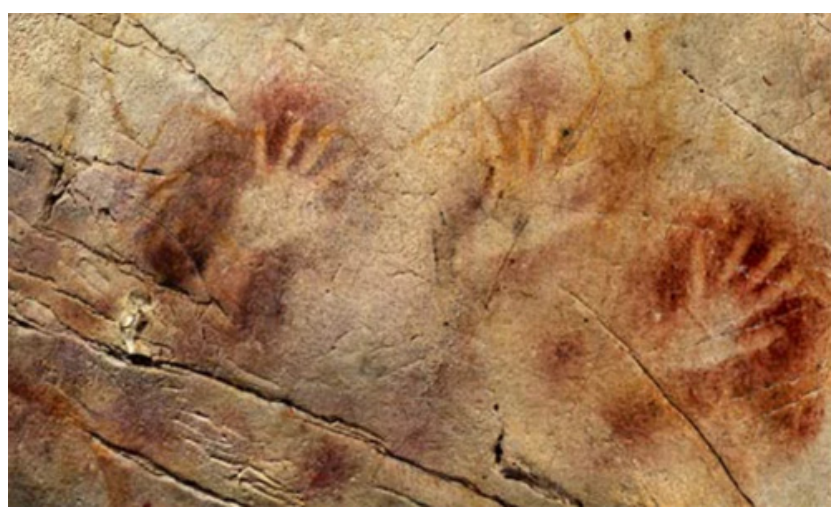

Fig. 1. Pinturas rupestres en la cueva El Castillo, que se estiman con una antigüedad de más de 37,000 años[1]

Se menciona en la literatura especializada [2], que las primeras imágenes construidas en el cerebro de los niños corresponden a manchas de color oscuro, ininteligibles entre sí y que por su naturaleza monocromática no permiten diferenciar claramente unos objetos de otros. Adicionalmente, parece que son tan sólo una representación bidimensional de la realidad percibida en las primeras etapas de vida de nuestra especie.

Curiosamente, desde hace unos años los códigos Quick Response se han convertido en una forma muy versátil de representación y almacenamiento de información, formándose, justamente, con las características que se han mencionado previamente y que a pesar de su extrema sencillez, logran ser un medio muy efectivo para transmitir y recibir información rápidamente: manchas negras bidimensionales e irreconocibles entre sí.

Estos casos constituyen solo dos ejemplos de la infinidad de representaciones que el hombre ha venido construyendo desde el inicio de su existencia: pasando por la que es quizás la pintura rupestre más antigua de la que se tiene noticia -cueva
El Castillo, España- [1] hasta llegar a los impresionantes efectos cinematográficos $3 \mathrm{D}-4 \mathrm{~K}$ que disfrutamos actualmente en las salas de cine; el ingenio humano siempre ha propendido por representar y comunicar de una manera impactante las construcciones que lleva a cabo dentro de su imaginación. Otrora, a través del virtuosismo y habilidad de sus propias manos; hoy día, con la ayuda de sendos paquetes informáticos que por medio de complejos algoritmos de programación, dan vida prácticamente a todo lo que se quiera recrear.

Claro está que la representación gráfica no ha tenido como único propósito las manifestaciones artísticas; en tiempos pretéritos, los grupos nómadas empleaban símbolos y trazos a manera de prevención en sitios inhóspitos, señalización de rutas y alerta de peligros. Estos símbolos irían evolucionando poco a poco hacia las primeras formas de escritura, los diferentes tipos de alfabetos que surgieron en el lejano oriente, y en la edad antigua y media, a la creación de elaboradas señales que orientaban a los viajeros en su periplo, siendo de eficaz ayuda para no perderse en los intrincados caminos de piedra de aquella época [3].

Más adelante y en otro campo del conocimiento, en el cenit del renacimiento verá la luz la obra de un personaje notable que dará un uso más "científico" a su trabajo gráfico: Andries van Wesel, más conocido como Andreas Vesalius, quien publica en 1543 De Humani Corporis Fabrica, cuyos dibujos en una obra en siete tomos resaltan la importancia de la disección y revelan detalles -hasta esos días inimaginables- de la verdadera estructura de la anatomía humana [4].

Siguiendo con esta línea de las ciencias naturales, enriquecida con la majestuosidad de los trazos a mano alzada, se tiene a Charles Darwin y su audaz boceto del árbol de la vida (Fig. 2, evolutionary tree). En efecto, este notable y profuso escritor inglés sentaría hacia 1837, en su primer cuaderno de notas sobre la transmutación de las especies y junto a su revolucionario dibujo, las bases de un sistema lógico que da explicación a la diversidad de las especies vivas [5].

Naturalmente, la práctica ingenieril no ha sido ajena a esta cadena de avances y aplicaciones en el campo de la representación gráfica. En un comienzo, se sabe que la civilización egipcia plasmó una 


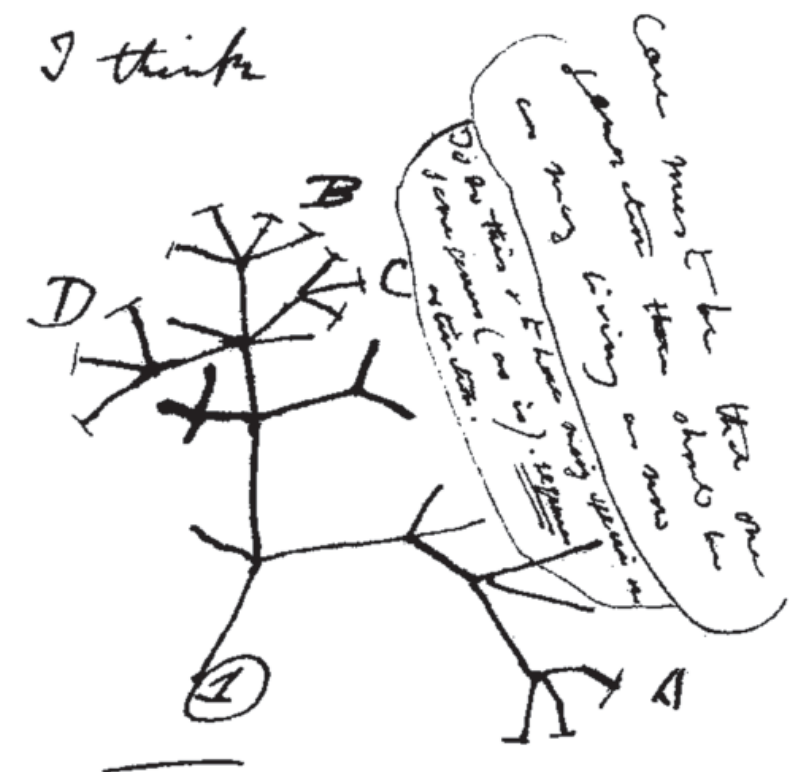

Fig.2. Primer esquema del árbol de la vida de Charles Darwin, de su Cuaderno B de la transmutación de las especies [5].

Las notas en manuscrito hablan de la relación entre extinción y número de individuos actuales para una especie.

robusta cantidad de conocimientos sobre geometría, hidráulica de ríos y métodos constructivos en el celebérrimo Papiro de Rhind -1650 A.C.- [6]; varios siglos después, en pleno apogeo del renacimiento italiano, el polifacético florentino Leonardo Da Vinci (1452-1519) creaba no sólo bocetos y fantásticas pinturas sobre inventos adelantados a su época; creó también las primeras maquetas y modelos a escala de puentes, torres y sistemas de defensa de ciudades hostigadas por ejércitos extranjeros[7].

\section{UNA NUEVA EXPERIENCIA DE DIBUJO}

Acercándonos al objeto central de este escrito y dando un salto de 500 años, a finales del milenio anterior hizo su entrada un software muy común en las empresas de arquitectura e ingeniería de nuestro tiempo: AutoCAD 2D-3D®. Miles de colores y texturas majestuosas, nuevos tipos de caligrafía inalcanzables para un díngrafo, la posibilidad de borrar una y otra vez sin temor a arruinar el papel; en suma, la panacea con la que soñaban cientos de delineantes al servicio de los departamentos de diseño.

Por supuesto que el ingenio de los programadores no se detuvo allí; un sinnúmero de paquetes de diseño asistido por computador hace su aparición año tras año desde el nacimiento de aquel icónico software. Surgieron Solid Edge ${ }^{\circledR}$, Rhinoceros ${ }^{\circledR}$, Adobe $C S \AA$ y otros más que permitieron dar rienda suelta a la creatividad del diseñador y que llevaron al dibujo a unos límites inimaginables, especialmente para aquellos quienes en los años ochenta todavía portaban los utensilios tradicionales para las clases de expresión gráfica y geometría descriptiva.

Por todo esto y en contraposición al enorme progreso computacional y las indiscutibles ventajas que ha traído a la industria la aplicación de este nuevo tipo de ayudas, surge la inquietud sobre la pertinencia de la enseñanza en el aula de los métodos clásicos de representación geométrica con los implementos de dibujo con que crecieron docenas de generaciones: una hoja en blanco, un lápiz, tal vez un par de escuadras y eso sí, la herramienta más perfecta con que cuenta el ser humano: sus manos.

No se está haciendo aquí una apología a los románticos postulados de los geómetras griegos, quienes echando mano de compás, regla e indudablemente una altísima dosis de imaginación, dieron solución a una gran cantidad de problemas matemáticos con estos elementos [8]. Se quiere es resaltar la importancia de una elaboración mental previa a las representaciones gráficas que se realizarán ya sea en un trozo de papel, ya sea en una computadora; y que sin duda, al pasar por la manipulación de unos instrumentos sencillos, enriquecerá y hará significativo el aprendizaje del estudiante, desarrollará habilidades manuales que serán las únicas con que contará en momentos específicos de su diario quehacer profesional y por último, no menos importante, puede explotar más a fondo todas las habilidades corporales y cognitivas con que él ha sido dotado. Fig. 3.

De hecho, una figura reconocible de la pedagogía americana contemporánea, David Ausubel, menciona en sus fundamentos del aprendizaje significativo que la interacción entre principios, procedimientos y normas derivará inevitablemente en un saber perdurable y que facilitará el recuerdo y la comprensión de los aspectos más importantes abordados en su proceso de aprendizaje [9]. Y son justamente estos tres elementos los pilares fundamentales -por citar un ejemplo- de un buen cro- 


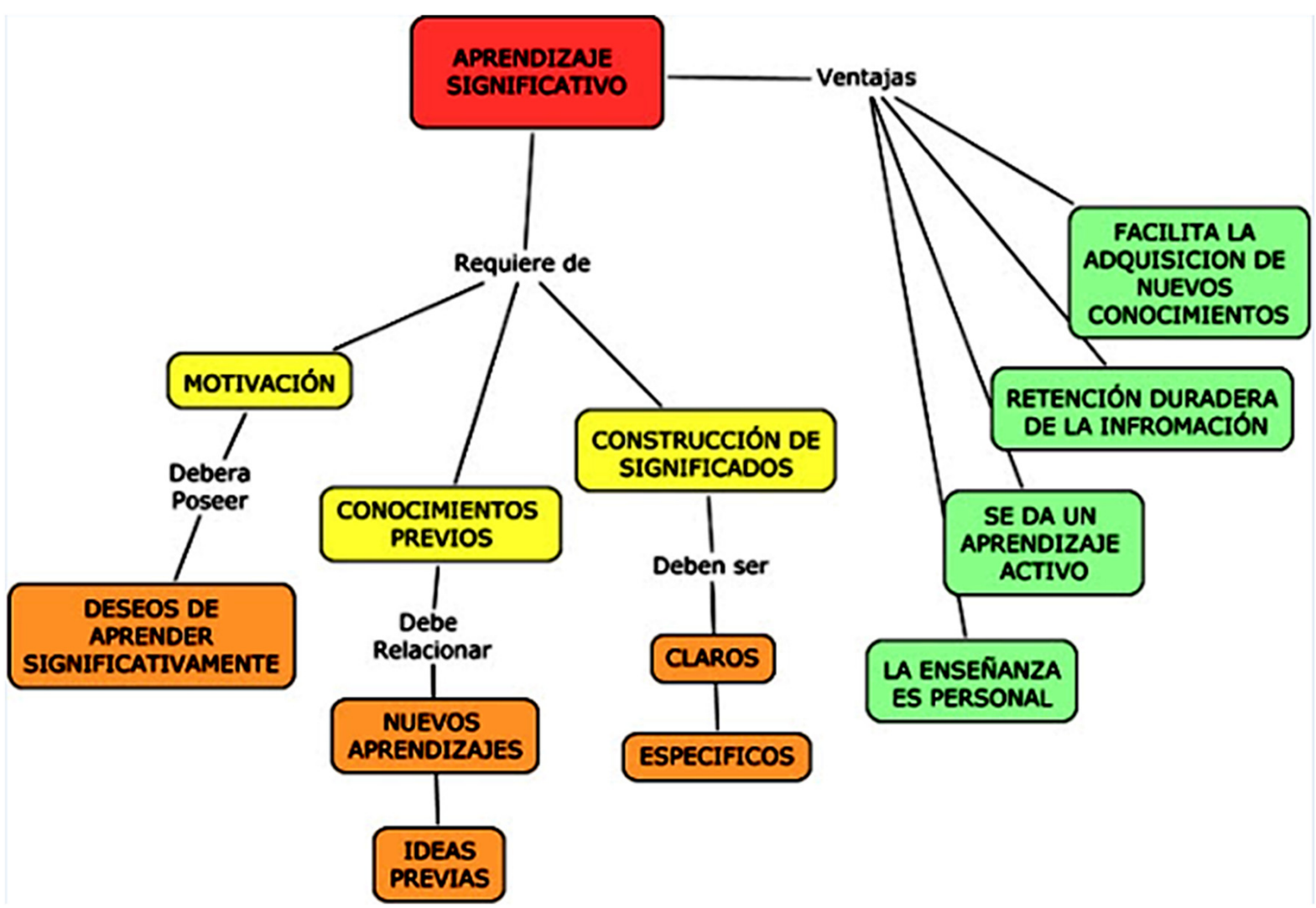

Fig. 3. Componentes del aprendizaje significativo, según Ausubel. Tomado de Lo que debe saber el docente de hoy". Blog de acceso libre.

quis que se haga con fines industriales: un plano que defina claramente el proceso constructivo, que esté sujeto a las normas de diseño y fabricación apropiados y que obedezca a la vez a los principios de viabilidad técnica y económica. Todo esto se puede lograr de seguro más rápido con la ayuda de una computadora. Incluso con alguna aplicación del dispositivo móvil. No obstante, en el aula, todo este proceso se verá ampliamente enriquecido cuando se involucre el uso de los instrumentos de dibujo manual y se incluya la escritura técnica, al menos a manera de esquema, en lo que será posteriormente un plano digitalizado en alguno de los programas disponibles en el amplio abanico comercial.

Por último, y para afianzar las tesis aquí expuestas, hay una condición inherente al ejercicio diario del ingeniero; muchas veces tendrá que dar soluciones a problemas que surgen súbitamente, en entornos donde no tendrá acceso inmediato a una computadora; ni siquiera tal vez a un escritorio o a una regla. Su capacidad de proyectar rápidamente un primer borrador de su solución, con unas dimensiones aproximadas y los materiales convenientes a emplear, esta capacidad, se ejercitará con mayor facilidad en la frecuente práctica de sus habilidades manuales y al entrar en contacto con el entorno que lo rodea. Es irrefutable el hecho que al sumergirse en contextos reales y al enfrentarse a problemas cotidianos, la capacidad creadora del ser humano se potencia mucho más que al estar en ambientes menos enriquecidos [10]. En esta dirección hay mucho por explorar en cuanto a la interacción Universidad Sociedad, objetivo misional de gran preponderancia para las instituciones públicas y privadas de educación superior.

\section{CONCLUSiOnes}

Como gran propuesta y a partir de lo expuesto anteriormente, a pesar del advenimiento de los grandes avances en el campo digital, no se puede separar de la práctica docente -en la enseñanza del dibujo técnico y otras asignaturas afines- el uso de los instrumentos tradicionales, la conceptualización 
de las construcciones básicas de geometría descriptiva, el trazo a mano alzada y el uso de una caligrafía clara y estética, como muestra de una formación integral de un profesional. Resulta evidente que este último tiene no solo en las matemáticas y la física el lenguaje por excelencia para transmitir sus conocimientos: la expresión gráfica hace parte esencial de su carrera y de su aporte a la continua construcción del conocimiento científico. No en vano, muchos de los grandes legados en materia de ciencia y tecnología han pasado por una buena ilustración o un brillante esbozo hecho a mano, tal como se expuso profusamente párrafos arriba. Para citar solo un ejemplo de enorme recurrencia en la ingeniería civil y mecánica, Sir Isaac Newton basó la demostración de sus tres leyes del movimiento en magníficas construcciones geométricas, que decoran una a una las páginas de su Principia Mathematica [11] realizada hacia 1687 en un estilizado latín y seguramente, con la ayuda de una arcaica regla y un pesado compás del Trinity College.

Para terminar y con el fin de presentar algunos trabajos realizados al interior de la Corporación Universitaria Republicana -Facultad de Ingeniería-, se muestra a continuación una galería fotográfica de algunos proyectos finales de la asignatura Dibujo técnico, orientada por el autor a un curso de primer semestre de la carrera de ingeniería industrial (Fig. 4). La foto grupal muestra al grupo de estudiantes y a sus amigos y familiares, que fueron invitados a la exposición de trabajos finales en 2017.

Adicionalmente, el autor ha sido realizador de programas de radio en la Universidad Nacional de Colombia, entre los que se cuenta el capítulo "Dibujos y bocetos como lenguaje tradicional", en el cual se hace una valiosa reflexión sobre la vigencia e importancia del dibujo manual en los tiempos actuales.

http:/ / unradio.unal.edu.co/nc/detalle/cat/ memoria-viva/article/dibujos-y-bocetos-como-lenguaje-tradicional-del-arquitecto.html

\section{REFERENCIAS}

[1] The Guardian. Neanderthals may have been first human species to create cave paintings. 2012.

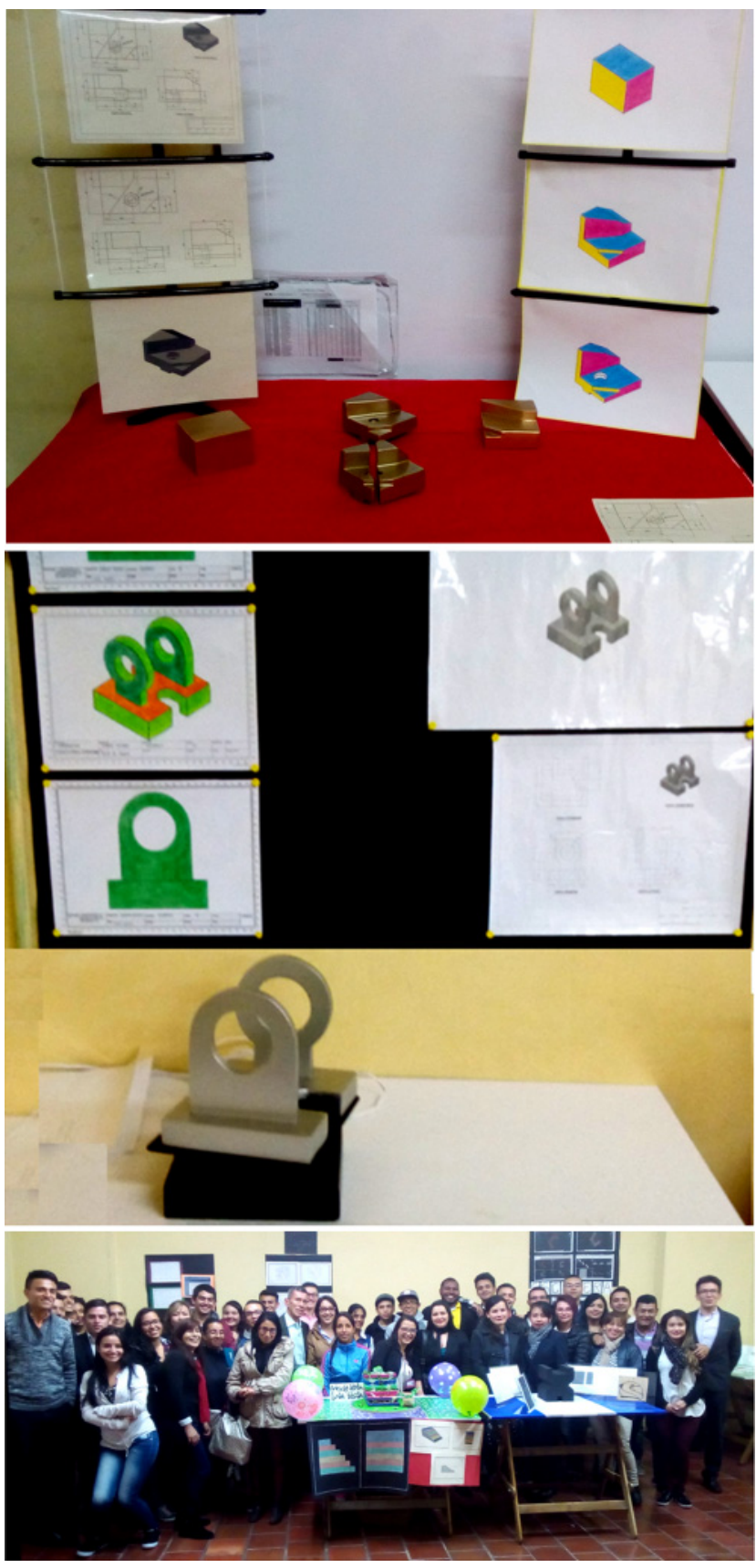

Fig. 4. Galería fotográfica de exposición de trabajos finales Dibujo Técnico, 2017. Corporación Universitaria Republicana [Archivo personal]

[2] A.A.O. Normal Vision Development in Babies and Children. American Academy of Ophthalmology. 2014.

[3] Fundación Universitaria del Área Andina: Historia, evolución y aplicaciones actuales de las señales. Documento del Taller de señalización - VI semestre. Documento digital en: https://es. calameo.com/read/004713862896abff648be 
[4] Richardson, WF (trad.). On the Fabric of the Human Body: A Translation of De corporis humani fabrica. San Francisco: Norman Publishing, 1998- (en curso). Vol. 1, 1998: ISBN 0-930405-73-0. Vol. 2, 1999: ISBN 0-930405-75-7. Vol. 3 y 4, 2003: ISBN 0930405-83-8.

[5] Van Wyhe, John. -. The Complete Work of Charles Darwin Online. ed. 2002. Edición digital en http:/ /darwin-online.org.uk/

[6] J. R. Newman, The world of mathematics. Mineola, New York: Dover Publications, INC. 2000.

[7] A. Vezzosi, Léonard de Vinci : Art et science de l'univers. Gallimard. 1996.
[8] S. Singh, The last theorem of Fermat. London: Four Estate Ltd. 1997.

[9] F. Díaz Barriga, Estrategias de enseñanza para la promoción de aprendizajes significativos. México: Mc. Graw Hill Education. 2010.

[10] L. Vygotski, Mind in society: The development of higher psychological. Cambridge, Massachusetts: Harvard University. 1978.

[11] I. Newton, Principia Mathematica. London: University of Glasgow. 1871. 